\title{
Overnight oximetry as a screening tool for moderate to severe obstructive sleep apnoea in South African children
}

\author{
M Zampoli, MB BCh, DCH, FC Paed, Cert Pulmonology (SA) Paed; N Abousetta, MB BCh, FC Paed (SA); \\ A Vanker, MB BCh, MMed (Paediatrics), FC Paed, Cert Pulmonology (SA) Paed, PhD \\ Division of Paediatric Pulmonology, Department of Paediatrics and Child Health, Red Cross War Memorial Children's Hospital and \\ Faculty of Health Sciences, University of Cape Town, South Africa
}

Corresponding author: M Zampoli (m.zampoli@uct.ac.za)

Background. Obstructive sleep apnoea (OSA) is common in children yet often overlooked, as symptom-based screening is unreliable. Polysomnography is regarded as the gold standard for the diagnosis of OSA, but is not widely available in South Africa (SA). Overnight oximetry is a validated screening tool for OSA.

Objectives. To describe the impact and utility of overnight oximetry at a tertiary children's hospital in SA.

Methods. A retrospective descriptive study was conducted of patients screened for OSA by overnight oximetry at a paediatric referral hospital from December 2012 to December 2014. Clinical data were retrieved from the oximetry database and medical records. Recordings of $\geq 6$ hours were considered adequate and included in the study. OSA severity was determined using the McGill score. Details on management and outcome were documented.

Results. Oximetry studies in 137 of 153 patients were suitable for analysis (88 males (64.2\%), median age 31.4 months (interquartile range (IQR) 15.8 - 65.8). Adenotonsillar hypertrophy was common ( $n=97,70.8 \%)$, and 65 children (47.4\%) had two or more underlying OSA risk factors. McGill's score classified patients as follows: no/mild OSA $n=55$ (40.1\%), moderate OSA $n=23$ (16.8\%), severe OSA $n=23$ (16.8\%) and very severe OSA $n=36$ (26.3\%). Male gender, adenotonsillar hypertrophy and a lower weight-for-age $z$-score $(-1.3 \mathrm{v}$. $-0.7 ; p=0.038)$ were associated with severe to very severe OSA. Seventy-eight children (56.9\%) were referred for surgery, 33 (24.1\%) receiving urgent surgery within a median of 6 days (IQR 4 - 12). In contrast, 59 children (43.1\%) with suspected OSA did not require surgical intervention.

Conclusions. Overnight oximetry is a simple low-cost tool to assess severity of OSA and prioritise appropriate OSA management in resource-constrained settings such as SA.

S Afr Med J 2019;109(1):23-26. DOI:10.7196/SAMJ.2019.v109i1.13033

Obstructive sleep apnoea syndrome (OSA) is a common condition in childhood, affecting 1 - 3\% of children, and can result in severe complications if left untreated. ${ }^{[1]}$ OSA is defined as a disorder of breathing during sleep characterised by prolonged partial upper airway obstruction or intermittent complete obstruction that disturbs normal ventilation during sleep and normal sleep patterns. ${ }^{[1]}$ While otherwise healthy children with adenotonsillar hypertrophy form the largest group of children with OSA, other high-risk groups include children with craniofacial malformations, anatomical narrowing of the upper airways, neuromuscular diseases or obesity, children who were premature babies and children with genetic disorders such as Down syndrome. ${ }^{[2]}$

A diagnosis of OSA is important in order to prevent complications of the condition, including failure to thrive, neurocognitive deficits, hyperactivity and cardiovascular sequelae such as pulmonary hypertension. Early diagnosis of OSA is important to guide appropriate treatment and interventions and to monitor the effectiveness of subsequent interventions. ${ }^{[2]}$

Adenotonsillectomy is recommended as first-line treatment of patients with symptomatic adenotonsillar hypertrophy. However, resources for this procedure are often scarce in resource-constrained settings, resulting in long surgical waiting lists. Prioritising patients who need urgent surgical intervention is therefore necessary. However, this is difficult without reliable objective evidence of OSA, as symptom-based questionnaire screening has been shown to be unreliable at predicting OSA..$^{[1,3]}$ Furthermore, knowledge of OSA severity is important for planning perioperative care and reducing perioperative complications. ${ }^{[4]}$

Screening for OSA symptoms is recommended at every routine health visit. This includes inquiring about snoring or symptoms of OSA and identification of underlying risk factors or medical conditions associated with OSA. ${ }^{[5]}$ Polysomnography (PSG) remains the recommended gold standard for the diagnosis of OSA ${ }^{[5]}$ However, PSG is expensive and labour intensive, requires specialised staff and is not available in resource-limited settings, including South Africa (SA). Ambulatory or in-hospital respiratory polygraphy sleep studies are gaining acceptability as an alternative to PSG. However, this technology also requires specialised equipment and expertise. ${ }^{[6,7]}$

The combination of overnight pulse oximetry and the McGill oximetry score (MOS) is a reliable screening tool for moderate to severe OSA. ${ }^{[4,8,9]}$ Overnight pulse oximetry is an accessible, simple and cost-effective alternative to PSG and may assist in prioritising children for operative interventions where resources are scarce.

\section{Objectives}

Red Cross War Memorial Children's Hospital (RCWMCH), a tertiary paediatric hospital in the Western Cape Province, SA, introduced overnight oximetry as a screening tool for OSA in 2012 with the aim of prioritising children requiring urgent intervention. We studied the spectrum of OSA severity and 'real-life' diagnostic utility of overnight oximetry as a tool for the diagnosis of OSA in children at RCWMCH. 


\section{Methods}

\section{Study design and setting}

A retrospective descriptive study was conducted of all patients 1 - 13 years of age screened with overnight oximetry for OSA by the pulmonology service at RCWMCH from 1 December 2012 to 31 December 2014. Approval to conduct the study was obtained from the Human Research Ethics Committee of the University of Cape Town (ref. no. 618/2015). Informed consent from participants was waived and permission to conduct the study was obtained from the hospital management.

Sleep study referral forms with check boxes containing relevant clinical information and an electronic results database of all oximetry studies performed during the study period were retrieved to identify eligible participants for the study. Children with cyanotic congenital heart disease, chronic lung diseases (excluding wellcontrolled asthma) and oximetry recordings with $<6$ hours of analysable data were excluded. In addition, only the first oximetry study was included if patients had more than one recording during the study period.

\section{Data collection}

Information relating to demographics, underlying comorbidity, symptoms as reported by caregivers, nutritional status (World Health Organization weight-for-age $z$-scores, body mass index) and likely cause(s) of OSA were documented by reviewing the medical records and referral form information.

\section{Overnight oximetry}

Patients with suspected OSA were admitted electively to a dedicated polysomnography room in a medical ward for unsupervised overnight oximetry. Oximetry studies performed in other wards of the hospital during hospitalisation were also included. Caregivers were required to stay with their child during the study and received a demonstration on how to operate the monitor.

Overnight oximetry was performed using portable Nonin 2500 (Nonin Medical Inc., USA) pulse oximeters, which have built-in data memory capacity of 48 hours of continuous recording with 4-second averaging intervals. Overnight recordings were downloaded via cable with nVision data management software (Nonin Medical Inc.). Graphical and tabulated automated oximetry trend reports were generated for each study.

\section{Classification of OSA severity with the MOS}

The MOS is a widely adopted tool that has been validated with polysomnography to classify OSA into no/mild, moderate or severe categories. Severity classification of OSA is based on the frequency and depth/nadir of the desaturations and the number of desaturation clusters, defined as periods of desaturation ( $\geq 5$ drops $\geq 4 \%$ from baseline) within a 10 - 30-minute period (Table 1). ${ }^{[4,8]}$ Results of overnight oxygen saturation trend graphs were manually analysed for quality and eligibility by the lead investigator (NA), who calculated the MOS.

\section{Treatment outcomes}

Medical records were reviewed to document which medical interventions were implemented after the overnight oximetry study. Interventions were classified as surgical or non-surgical. Non-surgical interventions included intranasal corticosteroids, antihistamines, leukotriene antagonists and non-invasive positivepressure ventilation. The time to surgery (in days) was recorded.

\section{Statistical analysis}

Data were analysed by standard statistical methods using Stata version 11 (StataCorp, USA). For descriptive statistics, continuous variables were expressed as means (standard deviations) for normally distributed variables or medians and interquartile ranges (IQRs). The Mann-Whitney $U$-test was used for comparing non-normally distributed measures and the $t$-test for normally distributed measures. Categorical variables were compared using the $\chi^{2}$ test.

\section{Results}

\section{Demographic and clinical characteristics}

A total of 153 oximetry recordings were identified, of which 137 were suitable for analysis. Demographics, reported symptom frequency and prevalence of underlying medical conditions or OSA risk factors are presented in Table 2. The median age was 31.4 months (IQR 15.8 65.8 ), with a greater number of males than females (64.2\% v. $35.8 \%)$. A history of snoring and parental concern about breathing during sleep were commonly reported ( $81.0 \%$ and $72.3 \%$, respectively). Adenotonsillar hypertrophy was the most common underlying risk factor for OSA ( $n=97,70.8 \%)$, and 65 children $(47.4 \%)$ had two or more underlying risk factors associated with OSA.

\section{OSA severity}

MOSs are presented in Fig. 1: 55 patients (40.1\%) had no/mild OSA, $23(16.8 \%)$ moderate OSA, 23 (16.8\%) severe OSA and 36 (26.3\%) very severe OSA. The median nadir oxygen saturation for mild to moderate OSA compared with severe to very severe OSA was $80 \%$ (IQR 65 - 84) v. 64\% (IQR 50 - 74) $(p<0.001)$. There were no significant differences in reported symptoms in children with

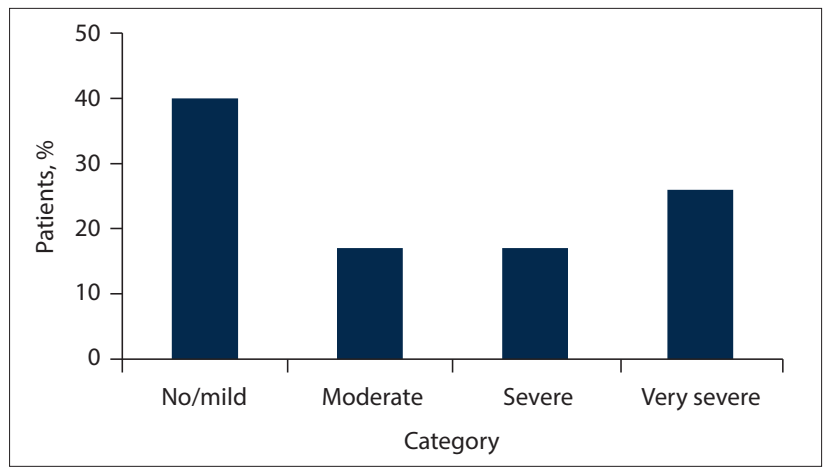

Fig. 1. Categories of obstructive sleep apnoea severity determined by the McGill oximetry score $(\mathrm{N}=137)$.

Table 1. McGill oximetry score and classification of OSA severity ${ }^{[4]}$

\begin{tabular}{llllll}
\hline McGill score & Desaturations $\leq \mathbf{9 0} \%, \boldsymbol{n}$ & Desaturations $\leq \mathbf{8 5} \%, \boldsymbol{n}$ & Desaturations $\leq \mathbf{8 0} \%, \boldsymbol{n}$ & Desaturation clusters, $\boldsymbol{n}$ OSA severity \\
\hline 1 & $<3$ & 0 & 0 & $<3$ & No or mild OSA \\
2 & $\geq 3$ & $\leq 3$ & 0 & $\geq 3$ & Moderate \\
3 & $\geq 3$ & $>3$ & $\leq 3$ & $\geq 3$ & Severe \\
4 & $>3$ & $>3$ & & & Very severe \\
OSA = obstructive sleep apnoea. & & & &
\end{tabular}


mild to moderate OSA compared with severe to very severe OSA. Male gender, adenotonsillar hypertrophy and a lower weight-for-age $z$-score $(-1.3$ v. $-0.7 ; p=0.038)$ were significant associations with severe to very severe OSA (Table 3 ).

\section{Interventional outcomes}

A new non-surgical intervention was initiated in response to the oximetry study in 52 children (38.0\%), 32/78 (41.0\%) with mild

\begin{tabular}{|c|c|}
\hline Variable & \\
\hline Patients, $N$ & 137 \\
\hline \multicolumn{2}{|l|}{ Demographic characteristics } \\
\hline Age (months), median (IQR) & $31.4(15.8-65.8)$ \\
\hline \multicolumn{2}{|l|}{ Gender, $n(\%)$} \\
\hline Male & $88(64.2)$ \\
\hline Female & $49(35.8)$ \\
\hline \multicolumn{2}{|c|}{ Clinical characteristics and reported symptoms } \\
\hline $\begin{array}{l}\text { Weight-for-age } z \text {-score, median (IQR) } \\
(n=126)\end{array}$ & $-0.9(-1.9-0.2)$ \\
\hline BMI, median (IQR) $(n=101)$ & $16.4(14.8-17.8)$ \\
\hline Snoring, $n(\%)$ & $111(81.0)$ \\
\hline Parental concern, $n(\%)$ & $99(72.3)$ \\
\hline Apnoea, $n(\%)$ & $77(56.2)$ \\
\hline Frequent awakenings, $n(\%)$ & $15(10.9)$ \\
\hline \multicolumn{2}{|l|}{ Underlying OSA risk factors } \\
\hline Adenotonsillar hypertrophy & $97(70,8)$ \\
\hline Neuromuscular disorders & $22(16.1)$ \\
\hline Facial skeletal abnormalities & $21(15.3)$ \\
\hline Obesity & $14(10.2)$ \\
\hline Down syndrome & $12(8.8)$ \\
\hline Prematurity & $13(9.5)$ \\
\hline Cerebral palsy & $10(7.3)$ \\
\hline
\end{tabular}

to moderate OSA and $20 / 59$ (33.9\%) with severe to very severe OSA. Seventy-eight children (56.9\%) were referred for surgery (mostly for adenotonsillectomy); 33 (24.1\%) required urgent surgical intervention, almost all $(30 / 33,90.9 \%)$ in the severe to very severe OSA group. The median time to urgent surgery in the severe to very severe group was 6 days (IQR 4 - 12). In contrast, 59 children (43.1\%) with suspected OSA did not require surgical intervention, 47/78 (60.2\%) with mild to moderate OSA and $12 / 59$ (20.3\%) with severe to very severe OSA. Non-invasive nocturnal ventilation or conservative management was initiated in children with severe or very severe OSA in whom adenotonsillectomy or tracheostomy was not indicated or appropriate.

\section{Discussion}

This study reports the initial 'real-life' experience and impact of implementing an overnight oximetry OSA screening protocol in a public sector referral hospital in SA. This study is novel, as oximetry is not widely available or practised in SA, where OSA diagnosis and management is an important but neglected child health concern. Furthermore, this study demonstrates the feasibility and clinical value of oximetry in settings without PSG to rapidly diagnose severe-spectrum OSA in children who need urgent intervention, which in our study was nearly half of all patients. As has been reported elsewhere, simple symptom-based screening in our study was unhelpful in distinguishing severe-spectrum OSA from less severe OSA, confirming the need for objective evaluation of children with suspected OSA in order to prioritise surgery for those who need it most. Equally importantly, oximetry identified non-severe OSA in over half of all referred children, thus sparing them unnecessary surgery and permitting prioritisation of more urgent cases.

The association of lower weight and adenotonsillar hypertrophy with severe OSA in our study confirms previously reported findings. A meta-analysis of studies measuring growth and growth biomarkers (e.g. insulin-like growth factor 1 and 3) before and after adenotonsillectomy showed significantly improved variables and growth after surgery. ${ }^{[10]}$ Clinicians should therefore consider

Table 3. Patient characteristics and severity of OSA $(N=137)$

\begin{tabular}{|c|c|c|c|}
\hline Variable & Mild/moderate & Severe/very severe & $p$-value \\
\hline Patients, $N(\%)$ & $78(56.9)$ & $59(43.1)$ & \\
\hline \multicolumn{4}{|l|}{ Patient characteristics } \\
\hline Age (months), median (IQR) & $34.4(14.9-69.3)$ & $30.6(16.9-58.9)$ & 0.823 \\
\hline Gender, $n(\%)$ & & & 0.011 \\
\hline Male & $43(55.1)$ & $45(76.3)$ & \\
\hline Female & $35(44.9)$ & $14(23.7)$ & \\
\hline Weight-for-age $\mathrm{z}$-score, median (IQR) $(n=126)$ & $-0.7(-1.7-0.5)$ & $-1.3(-2.3--0.4)$ & 0.038 \\
\hline BMI, median (IQR), $(n=101)$ & $16.9(14.9-21.5)$ & $16.0(14.8-17.2)$ & 0.195 \\
\hline Apnoea, $n(\%)$ & $39(50.0)$ & $38(64.4)$ & 0.092 \\
\hline Snoring, $n(\%)$ & $63(80.8)$ & $48(81.3)$ & 0.931 \\
\hline Frequent awakenings, $n(\%)$ & $9(11.6)$ & $6(10.2)$ & 0.799 \\
\hline Parental concern, $n(\%)$ & $52(66.7)$ & $47(79.7)$ & 0.093 \\
\hline Adenotonsillar hypertrophy, $n$ (\%) & $48(61.6)$ & $49(83.1)$ & 0.006 \\
\hline Neuromuscular disorders, $n(\%)$ & $12(15.4)$ & $10(16.9)$ & 0.805 \\
\hline Facial skeletal abnormalities, $n(\%)$ & $10(12.8)$ & $11(18.6)$ & 0.349 \\
\hline Obesity, $n(\%)$ & $11(14.1)$ & $3(5.1)$ & 0.096 \\
\hline Cerebral palsy, $n(\%)$ & $6(7.7)$ & $4(6.8)$ & 1.000 \\
\hline Down syndrome, $n(\%)$ & $9(11.6)$ & $3(5.1)$ & 0.232 \\
\hline Prematurity, $n(\%)$ & $6(7.7)$ & $7(11.9)$ & 0.409 \\
\hline
\end{tabular}


OSA when investigating children with unexplained poor growth. In contrast, obesity, a known risk factor for OSA, was not an important association in our study. However, the prevalence of obesity in this referred population was low, which may reflect referral patterns at our institution, which prioritised young children and children with other significant underlying OSA risk factors. It is unclear why children of male gender were more commonly referred in our study, or why male gender was associated with severe OSA. A higher prevalence of OSA in prepubertal males is less evident than in adults. ${ }^{[1]}$

Overnight oximetry and the MOS has been demonstrated in numerous studies to be a valid screening tool for detecting OSA, and its performance compared with PSG improves with increasing severity of OSA. ${ }^{[9]}$ In a landmark study by Brouillette et al., ${ }^{[12]}$ abnormal oximetry (MOS $\geq 2$ ) had a positive predictive value of 97\% in diagnosing PSG-confirmed OSA with an apnoea-hypopnea index (AHI) $>1$. However, sensitivity was low (43\%), indicating that a negative oximetry screen using MOS does not rule out OSA. Lowering the oximetry threshold (2 desaturation clusters and 1 desaturation event $<90 \%$ ) improves sensitivity and negative predictive values to $86 \%$ and $90 \%$, respectively. ${ }^{[13]}$ The oxygen desaturation index (ODI), a score that averages the number of desaturation events (drop of oxygenation saturation $\geq 4 \%$ from baseline) per hour may also be useful to screen for OSA. ${ }^{[9]}$ The MOS or ODI in combination with clinically based screening questionnaires can further improve the diagnostic accuracy of oximetry in OSA. ${ }^{[14-16]}$ More recently, the development of automated signal processing algorithms of remotely performed single-channel overnight oximetry recordings has shown high correlation with the AHI in habitually snoring children. ${ }^{[17]}$ Together with oximetry applications on smart mobile phones, advancements in technology will soon permit wide-scale low-cost ambulatory OSA screening and diagnosis. ${ }^{[18]}$

Oximetry has several limitations, however, including low sensitivity to diagnose mild-spectrum OSA and inability to distinguish obstructive from central apnoea or hypoventilation. Furthermore, the MOS has been based mostly on children with adenotonsillar hypertrophy who have a high pre-test probability of OSA, not children with underlying conditions associated with central apnoea or hypoventilation. Nevertheless, overnight oximetry can diagnose moderate to severe OSA accurately and allows prioritisation of scarce interventions for those children who need it most urgently. The childhood adenotonsillectomy trial (CHAT) has provided evidence for the benefit of adenotonsillectomy in mild OSA. ${ }^{[19]}$ The CHAT trial also documented a group of children who had complete resolution of PSG-diagnosed mild OSA which was predicted by a low baseline AHI and normal waist circumference, or low Pediatric Sleep Questionnaire and snoring scores. ${ }^{[20]}$ Watchful waiting with appropriate medical intervention in children with oximetry-diagnosed mild OSA is therefore an acceptable strategy in settings with long waiting lists for adenotonsillectomy.

\section{Study limitations}

Our study has several limitations. First, the retrospective design did not allow thorough or precise interrogation of the clinical presentation and symptoms at the time of oximetry studies. Second, the referred population in this cohort at a tertiary referral hospital is biased towards more severe and complex cases of OSA, and many of the patients had additional underlying OSA risk factors. The results of our study therefore do not represent otherwise healthy children with habitual snoring, which is more common in the general population. Lastly, owing to resource constraints and the study design, we could not report follow-up outcomes or repeat oximetry studies in those children with mild to moderate OSA who did not have surgery. It would be expected that some had persistent OSA that required further intervention.

\section{Conclusions}

The combination of overnight oximetry and the MOS is a feasible and useful means of diagnosing OSA and determining OSA severity in children with a wide range of underlying OSA risk factors and underlying diagnoses. Overnight oximetry should be widely adopted and implemented in SA as a low-cost tool to screen for OSA in children.

Declaration. None.

Acknowledgements. We thank Kirsty Britten for her assistance with data preparation and analysis.

Author contributions. MZ: conceptualised the study, supervised protocol development, collected and analysed data and wrote the manuscript; NA: developed protocol, collected and analysed data and approved the final manuscript; AV: conceptualised the study, supervised protocol development, collected and analysed data and approved the final manuscript.

Funding. None.

Conflicts of interest. None.

1. Spruyt K, Gozal D. Screening of pediatric sleep-disordered breathing: A proposed unbiased


discriminative set of questions

org/10.1378/chest.11-3164 Nixon GM, Brouillette RT. Sleep 8: Pae
https://doi.org/10.1136/thx.2003.007203

3. Spruyt K, Gozal D. Development of pediatric sleep questionnaires as diagnostic or epidemiological 3. Spruyt K, Gozal D. Development of pediatric sleep questionnaires as diagnostic or epidemiological
tools: A brief review of dos and don'ts. Sleep Med Rev 2011;15(1):7-17. https:///oi.org/10.1016/j. smrv.2010.06.003

4. Nixon GM, Kermack AS, Davis GM, Manoukian JJ, Brown KA, Brouillette RT. Planning adenotonsillectomy in children with obstructive sleep apnea: The role of overnight oximetry. Pediatrics 2004;113(1 Pt 1):e19-e25. https://doi.org/10.1542/peds.113.1.e19

5. Kaditis AG, Alonso Alvarez ML, Boudewyns A, et al. Obstructive sleep disordered breathing in 2- to 18-year-old children: Diagnosis and management. Eur Resp J 2016;47(1):69-94. https://doi. org/10.1183/13993003.00385-2015

6. Tan HL, Gozal D, Ramirez HM, Bandla HP, Kheirandish-Gozal L. Overnight polysomnography versus respiratory polygraphy in the diagnosis of pediatric obstructive sleep apnea. Sleep 2014;37(2):255-260. https://doi.org/10.5665/sleep.3392

7. Tan HL, Kheirandish-Gozal L, Gozal D. Pediatric home sleep apnea testing: Slowly getting there! Chest 2015;148(6):1382-1395. https://doi.org/10.1378/chest.15-1365

8. Nixon GM, Brouillette RT. Diagnostic techniques for obstructive sleep apnoea: Is polysomnography 8. Nixon GM, Brouillette RT. Diagnostic techniques for obstructive sleep apnoea: Is poly
necessary? Paediatr Respir Rev 2002;3(1):18-24. https://doi.org/10.1053/prrv.2002.0176

9. Kaditis A, Kheirandish-Gozal L, Gozal D. Pediatric OSAS: Oximetry can provide answers when Kaditis A, Kheirandish-Gozal L, Gozal D. Pediatric OSAS: Oximetry can provide answers when
polysomnography is not available. Sleep Med Rev 2016;27:96-105. https://doi.org/10.1016/j. polysomnography
smrv.2015.05.008

10. Bonuck KA, Freeman K, Henderson J. Growth and growth biomarker changes after adenotonsillectomy: Systematic review and meta-analysis. Arch Dis Child 2009;94(2):83-91. https://doi.org/10.1136/ adc.2008.141192

1. Brockmann PE, Koren D, Kheirandish-Gozal L, Gozal D. Gender dimorphism in pediatric OSA: Is it for real? Respir Physiol Neurobiol 2017;245(Nov):83-88. https://doi.org/10.1016/j.resp.2016.11.010

12. Brouillette RT, Morielli A, Leimanis A, Waters KA, Luciano R, Ducharme FM. Nocturnal pulse oximetry as an abbreviated testing modality for pediatric obstructive sleep apnea. Pediatrics 2000;105(2):405-412. https://doi.org/10.1542/peds.105.2.405

13. Velasco Suarez CT, Figueroa Turienzo JM, Len F, Mansilla E. Pulse oximetry recording in children with adenotonsillar hypertrophy: Usefulness in the diagnostic of obstructive sleep apnea syndrome. Arch Argent Pediatr 2013;111(3):196-201. https://doi.org/10.5546/aap.2013.eng.196

14. Pena-Zarza JA, Osona-Rodriguez de Torres B, Gil-Sanchez JA, Figuerola-Mulet J. Utility of the pediatric sleep questionnaire and pulse oximetry as screening tools in pediatric patients with suspected obstructive sleep apnea syndrome. Sleep Disord 2012;2012:819035. https://doi. org/10.1155/2012/819035

15. Chang L, Wu J, Cao L. Combination of symptoms and oxygen desaturation index in predicting childhood obstructive sleep apnea. Int J Pediatr Otorhinolaryngol 2013;77(3):365-371. https://doi. childhood obstructive sleep ap

16. Villa MP, Pietropaoli N, Supino MC, et al. Diagnosis of pediatric obstructive sleep apnea syndrome in seltings with limited resources. JAMA Otolaryngol Head Neck Surg 2015;141(11):990-996. https://doi.
s.tiln settings with limited resources. JAMA Otolaryngol Head Neck Surg 2015;141(11):990-996. https://doi.
org/10.1001/jamaoto.2015.2354

7ornero R, Kheirandish-Gozal L, Gutierrez-Tobal GC, et al. Nocturnal oximetry-based evaluation of habitually snoring children. Am J Respir Crit Care Med 2017;196(12):1591-1598. https://doi. org/10.1164/rccm.201705-0930OC

18. Garde A, Dehkordi P, Karlen W, Wensley D, Ansermino JM, Dumont GA. Development of a screening tool for sleep disordered breathing in children using the phone oximeter. PloS One 2014;9(11):e112959. https://doi.org/10.1371/journal.pone.0112959

9. Marcus CL, Moore RH, Rosen CL, et al. A randomized trial of adenotonsillectomy for childhood sleep apnea. N Engl J Med 2013;368(25):2366-2376. https://doi.org/10.1056/nejmoa1215881

20. Chervin RD, Ellenberg SS, Hou X, et al. Prognosis for spontaneous resolution of OSA in children. Chest 2015;148(5):1204-1213. https://doi.org/10.1378/chest.14-2873 